\title{
Uncertainties in microseismic event location analysis
}

\author{
Eryk Święch
}

AGH University of Science and Technology, Faculty of Geology, Geophysics and Environmental Protection, Department of Fossil Fuels; al. Mickiewicza 30, 30-059 Krakow; e-mail: swiech.eryk@gmail.com

(C) 2015 Authors. This is an open access publication, which can be used, distributed and reproduced in any medium according to the Creative Commons CC-BY 4.0 License requiring that the original work has been properly cited.

Microseismic monitoring is the observation technique of small earthquakes, called microseismic events, which occur in the ground as a result of human activities or industrial processes such as hydraulic fracturing.

Hydraulic fracturing is the one of the primary technologies for improving well productivity. The main purpose of hydraulic fracturing is to create an effective fracture system in the rock. During this process, fracturing fluids, which contain proppant, grains or sands, are pumped into a formation to hold the cracks open and keep oil and gas flow (Akram 2014). Microseismicity which occurs during the hydraulic fracturing process can be detected by sensitive sensors placed in a monitoring well nearby the treatment well (Pereira \& Jones 2010). The recording of microseismic events is very important. Identifying microseismicity distribution in hydraulic fracture treatments is crucial for deducing how the reservoir rocks are responding to the production activity and can be useful for evaluating the stimulation effectiveness in unconventional reservoirs for example by constraining a geomechanical model of fractured formation (Eisner et al. 2009). This is the reason why the correct location of microseismic events is a crucial factor in stimulation of unconventional reservoirs. One of the most important aspects in microseismic event location analysis is to investigate the uncertainties in these events location.

In this study, the author presents the application of different downhole array to investigate uncertainties in the estimated location of microseismic events. To achieve this goal, a 3D layered velocity model was used and several different receiver arrays were tested. All calculations were made in GeoTomo MiVu ${ }^{\mathrm{TM}}$ Microseismic Processing System. The author showed how the events location uncertainties changed with the location of the receiver array in a monitoring well.

This kind of analysis is very important prior to hydraulic fracturing process. It allows to confirm what kind of geometry should be used in a specified region to optimize the production process and to avoid errors caused by the ambiguity in microseismic data interpretation.

\section{REFERENCES}

Akram J., 2014. Downhole microseismic monitoring: processing, algorithms and error analysis. Department of $\mathrm{Ge}-$ oscience, Calgary, Alberta.

Eisner L., Duncan P.M., Heigl W.M. \& Keller W.R., 2009. Uncertainties in passive seismic monitoring. The Leading Edge.

Pereira A.M. \& Jones M., 2010. Fundamentals of borehole seismic technology. Schlumberger. 